\title{
An unusual case of positive sIgE to Galactose-alpha- 1,3-galactose from South Italy
}

${ }^{1}$ Allergy Diseases Center Prof G. Bonsignore, Institute of Biomedicine and Molecular Immunology A. Monroy (IBIM), National Research Council (CNR), Palermo, Italy

${ }^{2}$ Laboratorio di Entomologia e Controllo Vettori Ambientali, Istituto Zooprofilattico Sperimentale della Sicilia A. Mirri, Italy ${ }^{3}$ C.Re.N.A. (Centro di Referenza Nazionale Anisakiasi), Istituto Zooprofilattico Sperimentale della Sicilia A. Mirri, Italy ${ }^{4}$ Clinical Pathology, Allergy Unit, Buccheri La Ferla Hospital, Palermo, Italy

The work was carried out both at Laboratorio di Entomologia e Controllo Vettori Ambientali, Istituto Zooprofilattico Sperimentale della Sicilia A. Mirri and at Clinical Pathology, Allergy Unit, Buccheri La Ferla Hospital, Palermo, Italy

\section{KEY WORDS}

sIgE to $\alpha$-Gal; delayed food

anaphylaxis; tick bit; basophil

activation test; hunter

\section{Corresponding author}

Carina G. Uasuf

Via Ugo La Malfa 153

90146 Palermo, Italy

E-mail: cuasuf@gmail.com

Phone: +393395975737

Fax: +390916809122

\section{Doi}

10.23822/EurAnnACI.1764-1489.25

\begin{abstract}
Summary
We report the case of a 38-year-old man who was bitten several times during his life by a tick. He didn't report any previous history of anaphylaxis after the ingestion of red meat. The serum specific IgE showed positivity to $\alpha-G a l$. The proximity of the bits didn't increase the titer of IgE antibodies to alpha-gal. We could hypothesize that the frequency of the exposure to the tick bites and the amount of tick bites during his lifetime induced a sort of tolerance in this patient.
\end{abstract}

\section{Introduction}

A new form of delayed anaphylaxis has been observed in the United States since 2009 (1), due to IgE antibody directed at a mammalian oligosaccharide epitope, galactose-alpha-1,3-galactose $(\alpha-\mathrm{Gal})$.

This antibody was recently identified in two subsets of patients: a, patients who had developed severe anaphylactic reactions at the first treatment with cetuximab (2); and b, patients who had developed delayed-onset anaphylaxis 3-6 hours after the ingestion of red meat.

Further studies strongly suggested that tick bites were a cause, if not the only significant cause, of $\operatorname{IgE~Ab}$ responses to $\alpha-\mathrm{Gal}$ in the United States (3). Subsequently, in Australia (4) and in
Europe (5) cases of delayed anaphylaxis related to IgE-mediates alpha-gal sensitisation have been described, although the tick responsible seems to be Ixodes in Europe, while Amblyoma americanum seems to be the vector in the USA.

Recently, in Italy both allergy (6) and a high prevalence of sIgE to Galactose-alpha-1,3-galactose have been described in rural areas (7). Therefore, we've decided to investigate if this oligosaccharide could be detected in South Italy.

\section{Material and methods}

We report a case of a 38-years-old man who was bitten by a tick on December 2016 and went to the Zooprophylactic institute of Palermo to control if Rickettsia infection developed. 
A serum sample and an interview were obtained from this man, that was a veterinarian from Bisacquino, a little town placed at 710 meter above the sea, in West Sicily. He told us that while he was hunting, he was bitten by an insect that he recognized as a tick. He brought the insect to the Zooprophylactic institute for identification, and they confirmed that the insect was a tick from the Ixodes ricinus species. This type of tick is frequently found in the hunting area of Bisacquino.

Immediately, they analyzed the serum for antibodies to Rickettsia, and the result was negative.

A detailed clinical history was collected. The patient used to hunt 3 times a year. He reported that he was bitten by a tick 3 times / year minimum for almost 20 years during his lifetime. As usually, last year he was bitten 3 times (once in January, twice in December with an interval of 20 days) and once in January 2017.

Surprisingly, he had been eating every kind of meat (pork, beef, lamb, chicken, rabbit, liver meat, etc) after all episodes of tick bit, without developing any early or delayed allergic symptoms. No previous history of anaphylaxis after the ingestion of red meat was reported. No history of malignancy in therapy with cetuximab or other similar was reported. Only a positive history of seasonal rhinitis was reported in the last year.

ImmunoCap technique (Thermofisher, Sweden) was used to measured sIgE antibodies to $\alpha-\mathrm{Gal}$ in the sera of the patient collected on December 2016 (20 days after the first tick bite in that month) and 1 day after the last tick bit (January 2017). For $\operatorname{sIgE}$, the cut-off used for a positive reaction was $\geq 0.1 \mathrm{kU}_{\mathrm{A}} / \mathrm{L}$ as suggested by the manufacturer. Surprisingly, the results were $1.08 \mathrm{kU}_{\mathrm{A}} / \mathrm{L}$ and $0.58 \mathrm{kU}_{\mathrm{A}} / \mathrm{L}$, respectively. sIgE for pork, beef, lamb and milk were negative. sIgE for inhaled allergens were positive only for Dermatophagoides pteronyssinus $1.73 \mathrm{kU}_{\mathrm{A}} / \mathrm{L}$, Parietaria $16.7 \mathrm{kU}_{\mathrm{A}} / \mathrm{L}$ and Cupressus $1.82 \mathrm{kU}_{\mathrm{A}} / \mathrm{L}$. Dermatophagoides farinae, Graminacea mix, olive, cat, dog and Alternar$i a$, were negative. Total $\mathrm{IgE}$ was $53.8 \mathrm{kU} / \mathrm{L}$. The day after the last tick bit, basophil activation test with beef and cetuximab in different dilution was performed. Interesting, the results of the basophil activation with both allergens were below the cut-off

Figure 1a - \% of CD 63 activated basophils with beef.

Beef

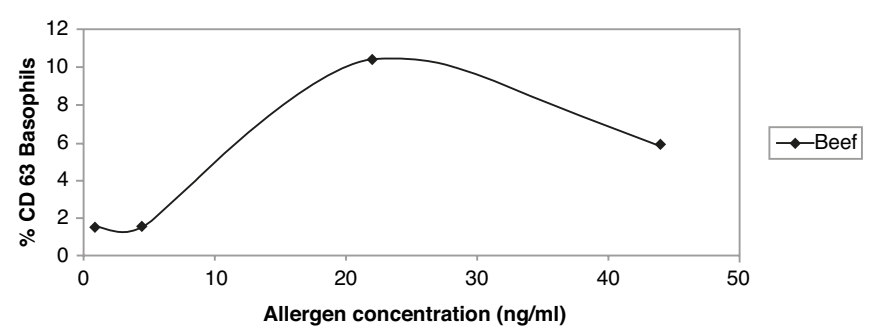

suggested by the manufacturer ( $\geq 15 \%$ of activated basophils for food, and $\geq 5 \%$ for drugs). (Figure 1a, 1b)

\section{Discussion}

To our knowledge, this is the first time that a case of positive $\alpha$-Gal patient without symptoms has been reported in Italy. It has been described that the $\operatorname{IgE} A b$ to $\alpha-G a l$ appears to decrease over time, but this trend can be reversed by additional tick bites (8). Thus, patients can be led to believe that they are no longer allergic to mammalian meat because they have eaten small amounts of meat without reactions (likely, their $\operatorname{IgE} \mathrm{Ab}$ to $\alpha$-Gal has fallen quite low). Overall, the factors which feed into the equation to produce a reaction are clearly complex and variable, especially in the setting of an $\operatorname{IgE} A b$ to $\alpha$-Gal that may 'naturally' decrease over time (9).

In this patients, the risk factors like number of tick bites during the lifetime and recent tick bites did not show a higher value of positivity, as it has been described by Villalta et al. (7). On the other hand, we agree with this author with respect to the risk factors associated with increased sIgE to $\alpha$-Gal, like atopy (this patient suffer from a poly-sensitized allergic rhinitis), male gender and hunting.

Interesting, the results of the basophil activation test (below the cut-off for beef and cetuximab) correlate with the absence of clinical symptoms in this patient.

Until today, this patient hasn't developed any type of allergic reaction after eating any type of red meat. Very interestingly, the proximity of the bits hasn't increased the titer of $\operatorname{IgE}$ antibodies to alpha-gal.

Allergy is not a disease in itself, but a mechanism leading to disease that not always is clinically manifested. Sensitization is a complex interplay among the individual exposed (inherited risk of becoming allergic), the timing of exposure, the dose, the context of exposure and endogenous properties of the allergen (10). Therefore, we could hypothesize that the frequency (minimum 3 times / year) of the exposure to the tick bites and the amount of tick bites during his lifetime have induced a sort of tolerance in this patient.

Figure $\mathbf{1 b}$-\% of CD 63 activated basophils with cetuximab.

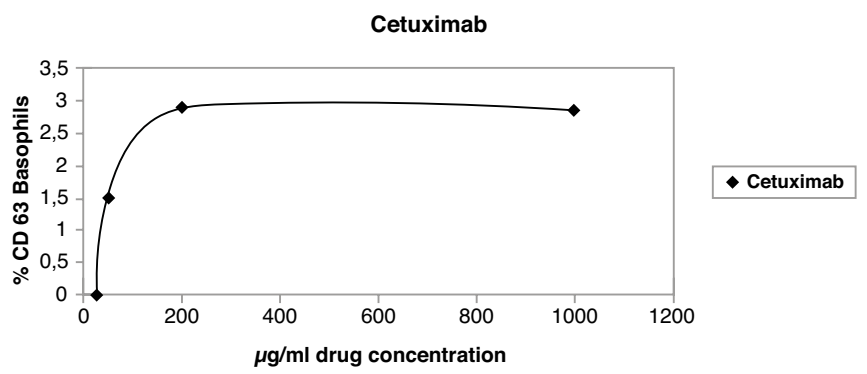




\section{Conclusion}

The evidence about ticks as a cause of the IgE response is good, but certainly it does not exclude a role for other parasitic exposures (11).

Why some positive $\alpha$-Gal patients develop delayed-onset anaphylaxis after the ingestion of red meat and others do not, is a question that needs to be answered. Immunological studies need to be done to clarify this.

\section{Conflict of interest}

The authors declare that they have no conflict of interest.

\section{References}

1. Commins SP, Platt-Mills TA Anaphylaxis syndrome related to a new mammalian cross-reactive carbohydrate determinant. J Allergy Clin Immunol 2009; 124(4):652-7.

2. Chung $\mathrm{Ch}$, Mirakhhur B et al. Cetuximab-induced anaphylaxis and IgE specific for galactose-alpha-1,3-galactose. N Engl J Med 2008; 358(11):1109-17.

3. Commins SP, Platts-Mills TA Tick bites and red meat allergy. Curr Opin Allergy Clin Immunol 2013; 13(4):354-9.
4. Van Nunen SA, O'Connor KS et al. An association between tick bite reactions and red meat allergy in humans. Med J Aust 2009; 190(9):510-1.

5. Nunez R, Carballada F et al. Delayed mammalian meat-induced anaphylaxis due to galactose in 5 European patients. J Allergy Clin Immunol 2011; 128(5):1122-3.

6. Calamari AM, Poppa M, Villalta D, Pravettoni V. Alpha-gal anaphylaxis: the first case report in Italy. Eur Ann Allergy Clin Immunol. 2015; 47(5):161-2.

7. Villalta D, Pantarotto L, Da Re M, Conte M, Sjolander S, Borres MP, Martelli P. High prevalence of sIgE to Galactose- $\alpha-1,3$-galactose in rural pre-Alps area: a cross-sectional study. Clin Exp Allergy. 2016; 46(2):377-80. doi: 10.1111/cea.12655.

8. Commins SP, James HR, Kelly LA, et al. The relevance of tick bites to the production of $\mathrm{IgE}$ antibodies to the mammalian oligosaccharide galactose- $\alpha-1,3$-galactose. J Allergy Clin Immunol 2011; 127:1286-93. e1286.

9. Commins SP, Thomas A, Platts-Mills E. Delayed anaphylaxis to red meat in patients with IgE specific for galactose alpha-1,3-galactose (alpha-gal). Curr Allergy Asthma Rep 2013; 13(1):72-7.

10. van Ree R. Allergens - structure and function. Global Atlas of Allergy 2014; 6-8.

11. Thomas A.E. Platts-Mills, Alexander J. Schuyler, Anubha Tripathi, and Scott P. Commins. Anaphylaxis to the Carbohydrate Side-Chain Alpha-gal. Immunol Allergy Clin North Am 2015; 35(2):247-60. doi:10.1016/j.iac.2015.01.009. 